\title{
Experimental and theoretical study of rapid flows of cohesionless granular materials down inclined chutes
}

\author{
N. P. Kruyt and W. J. Th. Verël \\ Laboratory for Bulk Solids Handling, Department of Mechanical Engineering, University of Twente, P.O. Box 217, 7500 AE Enschede \\ (Netherlands)
}

(Received January 20, 1991; in revised form June 23, 1992)

\begin{abstract}
A theoretical and experimental study is performed of rapid, fully developed flows of cohesionless granular materials down inclined chutes with a rough base. Two flow types are studied in detail: (1) immature sliding flow, where a stagnant zone forms on the base of the chute, and (2) fully developed sliding flow, where no such zone is formed. A simple phenomenological theory is developed that predicts the flow type and the associated velocity profile. The theory models dynamic stresses induced by interparticle collisions as well as quasi-static stresses induced by friction acting on semi-permanent interparticle contacts. Hence it is developed for the so-called frictional-collisional regime. Employing a photographical method, the flow type and the velocity profile are determined experimentally for various chute angles in a test set-up in which the granular material is continuously circulated. Quantitative agreement between the theoretical and the measured velocity profile is reasonably good, although it appears that at the largest chute angle the side wall friction causes deviations between the theoretical and the measured velocity profile.
\end{abstract}

\section{Introduction}

Rapid flows of cohesionless granular materials down inclines occur in various industrial situations, such as in hoppers of silos and in other bulk solids handling equipment, as well as in various geophysical situations. In order to study constitutive relations for cohesionless granular materials valid under these conditions, a theoretical and experimental study has been performed of rapid flows of cohesionless granular materials down inclined chutes with a rough base.

A review of rapid granular flows, including the flow down inclined chutes, is given in [1]. Many recent theoretical studies are based on computer simulation of the behaviour of the assemblies of particles and on micro-structural theories that incorporate elementary properties of the particles, such as coefficient of restitution, interparticle friction, etc. A recent review of these micro-structural theories is given in [2].

Ishida et al. [3] discerned three types of flow down inclined chutes with a rough base. These three types, listed in the order they occur when the chute angle is increased, are:

(a) Immature sliding flow:

This type of flow occurs when the chute angle is close to the quasi-static friction angle of the granular material. A stationary layer of material forms on the base of the chute, thicker on the upstream than on the downstream side. The velocity profile has an inflection point.

(b) Fully developed sliding flow:

If the chute angle is increased the stationary layer disappears, and the velocity profile becomes blunter (fuller) in shape. The flow approaches the graininertia regime where the stresses are mainly determined by collisions between the particles. Saltation of particles near the free surface is present.

(c) Splashing flow:

The velocity profile becomes even blunter and saltation is vigorous; a cloud of saltating particles exists instead of a clearly defined free boundary.

The types of chute flow that will be considered in the sequel are the immature sliding flow and the fully developed sliding flow, which correspond to the socalled frictional-collisional regime. These will be studied experimentally and theoretically.

\section{Stress field}

The flow of cohesionless granular material down an inclined chute of a finite width $W$, as shown in Fig. 1, is considered here. The velocity of the granular material is assumed zero on the base of the chute; this means 


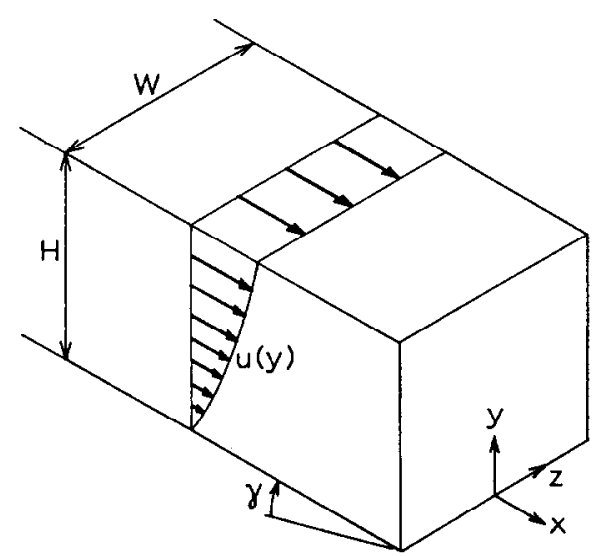

Fig. 1. Geometry of the chute.

that it is perfectly rough. The angle of the chute with the horizontal is denoted by $\alpha$. It is assumed that the flow depth $H$ of the granular material is constant. The angle of this flowing layer with the horizontal is denoted by $\gamma$. Depending on chute angle, material properties, side wall friction, chute geometry and mass flow rate, a stagnant layer of material may form in the chute. If present, the angle of this stagnant layer with the horizontal is denoted by $\beta$. It is clear that $\gamma$ is given by:

$\gamma=\max (\alpha, \beta)$

Immature sliding flow corresponds to $\gamma=\beta$, while $\gamma=\alpha$ corresponds to fully developed sliding flow.

It is assumed that the flow is a fully developed shear flow and hence the (continuum) velocity field is: $u=(u(y), 0,0)^{\mathrm{T}}$. For the sake of simplicity, the bulk density $\rho$ of the material is taken to be constant. The effect of the interstitial air can be neglected when the material consists of relatively coarse particles. Tensile stresses are reckoned positive, this being the usual convention in continuum mechanics. The stress tensor is denoted by $T$.

With these assumptions, the momentum equations become:

$\frac{\partial}{\partial y} T_{x y}+\frac{\partial}{\partial z} T_{x z}=-\rho g \sin \gamma$

$\frac{\partial}{\partial y} T_{y y}=\rho g \cos \gamma$

$$
\frac{\partial}{\partial z} T_{z z}=0
$$

Note that $T_{x z}$ is not neglected here in order to incorporate the effect of the side wall friction. This effect is included in the determination of the stress field in the chute in the manner of [4]: it is assumed that the side walls are smooth enough that slips occur and that the flow field remains two-dimensional but that the stress field is three-dimensional with shear stresses existing on planes parallel to the vertical $x-y$ plane. The shear stress $T_{x z}$ is approximated by:

$T_{x z} \cong 2 T_{x z}^{\mathrm{w}} \frac{z}{W}$

where

$\left|T_{x z}^{\mathrm{w}}\right|=\tan \epsilon\left|T_{z z}^{\mathrm{w}}\right|$

Here, $\epsilon$ is the friction angle of the side wall. The stresses $T_{z z}^{\mathrm{w}}$ and $T_{y y}^{\mathrm{w}}$ at the side wall are related by a stress ratio $k$ :

$T_{z z}^{\mathrm{w}}=k T_{y y}^{\mathrm{w}}$

At the top of the flowing layer of granular material $y=H$ the boundary conditions are:

$T_{x y}=0$ and $T_{y y}=0$

From (3) and (8) it follows that $T_{y y}$ is given by:

$T_{y y}=-\rho \mathrm{g} H \cos \gamma\left(1-\frac{y}{H}\right)$

and $T_{x y}$ is determined from (2), (5), (6), (7), (8) and (9):

$T_{x y}=\rho g h \cos \gamma\left(1-\frac{y}{H}\right)\left[\tan \gamma-k \tan \epsilon \frac{H}{W}\left(1-\frac{y}{H}\right)\right]$

If present, the angle $\beta$ of the stationary layer of material in the chute with the horizontal is determined from the condition that at $y=0$ :

$\left|\frac{T_{x y}}{T_{y y}}\right|=\tan \phi_{\mathrm{s}}$

Here $\phi_{\mathrm{s}}$ is the quasi-static friction angle of the material. From (9), (10) and (11) it follows that:

$\tan \beta=\tan \phi_{\mathrm{s}}+k \tan \epsilon \frac{H}{W}$

\section{Constitutive relation}

Micro-structural studies based on kinetic theory (see [2] for a recent review) have greatly increased the understanding of the behaviour of cohesionless granular materials under high shear-rate conditions. In these studies interparticle collisions form the dominant mechanism of momentum transfer. In the chute flows considered here, not only the dynamic stresses induced by these collisions are important, but also the quasi-static stresses associated with frictional forces acting on semi- 
permanent interparticle contacts. These dynamic and quasi-static stresses are related to the total stress. In [5] and [6] a linear relation between quasi-static stress $T^{\mathbf{S}}$, dynamic stress $\boldsymbol{T}^{\mathrm{D}}$ and total stress $T$ was proposed. The phenomenological relation proposed here is inspired by the Casson model [7]:

$\left(T_{x y}\right)^{1 / 2}=\left(T_{x y}^{\mathrm{s}}\right)^{1 / 2}+\left(T_{x y}^{\mathrm{D}}\right)^{1 / 2}$

and

$\left(-T_{y y}\right)^{1 / 2}=\left(-T_{y y}^{\mathrm{s}}\right)^{1 / 2}+\left(-T_{y y}^{\mathrm{D}}\right)^{1 / 2}$

The dynamic stresses are modelled in accordance with [8]: both dynamic shear stress and dynamic normal stress are proportional to the square of the shear-rate $\mathrm{d} u / \mathrm{d} y$.

$T_{x y}^{\mathrm{D}}=\mu\left(\frac{\mathrm{d} u}{\mathrm{~d} y}\right)^{2}$

$\left|\frac{T_{x y}^{\mathrm{D}}}{\mathrm{T}_{y y}^{\mathrm{D}}}\right|=\tan \phi_{\mathrm{D}}$

In (16) $\phi_{\mathrm{D}}$ denotes the dynamic friction angle. Tests with dry granular materials in annular shear cells have shown, [9], (10], [11], that $\phi_{\mathrm{D}}$ is only weakly dependent upon solid concentration and shear-rate. According to [8], $\mu$ is proportional to the density of the particles and the square of the particle radius.

Similar to [5] and [6] it is assumed here that the quasi-static shear stress is proportional to the quasistatic normal stress:

$\left|\frac{T_{x y}^{\mathrm{s}}}{T_{y y}^{\mathrm{s}}}\right|=\tan \phi_{s}$

From (13), (14), (15), (16) and (17) it follows:

$$
\begin{aligned}
\frac{\mathrm{d} u}{\mathrm{~d} y}= & \frac{1}{\left(\tan \phi_{\mathrm{D}}\right)^{1 / 2}-\left(\tan \phi_{\mathrm{s}}\right)^{1 / 2}} \\
& \times\left(\frac{\tan \phi_{\mathrm{D}}}{\mu}\right)^{1 / 2}\left(\left(T_{x y}\right)^{1 / 2}-\left(-\tan \phi_{\mathrm{s}} T_{y y}\right)^{1 / 2}\right)
\end{aligned}
$$

and

$$
\begin{aligned}
-T_{y y}^{\mathrm{s}}= & \frac{1}{\left\{\left(\tan \phi_{\mathrm{D}}\right)^{1 / 2}-\left(\tan \phi_{\mathrm{s}}\right)^{1 / 2}\right\}^{2}} \\
& \times\left(\left(-\tan \phi_{\mathrm{D}} T_{y y}\right)^{1 / 2}-\left(T_{x y}\right)^{1 / 2}\right)^{2}
\end{aligned}
$$

From (9), (10), (19) and neglecting the effect of the side wall friction, it follows that $T_{y y}^{s}$ becomes positive for $y=0$ when $\alpha>\phi_{\mathrm{D}}$. This is physically impossible, since a cohesionless granular material cannot sustain tensile stresses. The interpretation is that fully developed, non-accelerating flows are only possible for $\alpha<\phi_{\mathrm{D}}$.

\section{Theoretical velocity profile}

The velocity profile corresponding to the proposed constitutive relation will be determined here. Substitution of (9) and (10) into (18) gives the velocity gradient:

$\frac{\mathrm{d} u}{\mathrm{~d} y}=C\left(1-\frac{y}{H}\right)^{1 / 2}\left(\left(A+B \frac{y}{H}\right)^{1 / 2}-1\right)$

where

$C=\frac{1}{\left(\tan \phi_{\mathrm{D}}\right)^{1 / 2}-\left(\tan \phi_{\mathrm{s}}\right)^{1 / 2}}\left(\frac{\tan \phi_{\mathrm{s}} \tan \phi_{\mathrm{D}} \rho g H \cos \gamma}{\mu}\right)^{1 / 2}$

$A=\frac{\tan \gamma-k \tan \epsilon \frac{H}{W}}{\tan \phi_{\mathrm{s}}}$

$B=\frac{k \tan \epsilon \frac{H}{W}}{\tan \phi_{\mathrm{s}}}$

From (12) it follows that immature sliding flow $(\gamma=\beta)$ is characterised by $A=1$, while for fully developed sliding $(\gamma=\alpha) A>1$. Parameter $B$ is a measure of the effect of the side-wall friction.

Integration of (20) with the no-slip condition at $y=0$ gives the theoretical velocity profile:

$$
\begin{aligned}
u(y)= & C H\left[\frac { ( A + B ) ^ { 2 } } { 4 B \sqrt { B } } \left\{\arctan \left(\frac{B}{A}\right)^{1 / 2}\right.\right. \\
& \left.-\arctan \left(\frac{B-B \frac{y}{H}}{A+B \frac{y}{H}}\right)^{1 / 2}\right\}+\left\{\frac{2}{3}-\frac{1}{2}\left(A+B \frac{y}{H}\right)^{1 / 2}\right\} \\
& \times\left(1-\frac{y}{H}\right)^{3 / 2}+\frac{A+B}{4 B}\left(A+B \frac{y}{H}\right)^{1 / 2} \\
& \left.\times\left(1-\frac{y}{H}\right)^{1 / 2}-\frac{3 A \sqrt{A}-3 \sqrt{A} B+8 B}{12 B}\right]
\end{aligned}
$$

The shape of the dimensionless velocity profile is depicted in Figs. 2, 3 and 4 for various values of the parameters $A$ and $B$. For $A=1$ the velocity profile has an inflection point. When the parameter $A$ is increased, as is the case when the chute angle $\alpha$ is increased, the shape of the velocity profile becomes blunter in shape. These findings are in qualitative agreement with the observations of [1]. The presented theory predicts the transition from immature sliding flow to fully developed sliding flow, and the associated change in the shape of the velocity profile. 


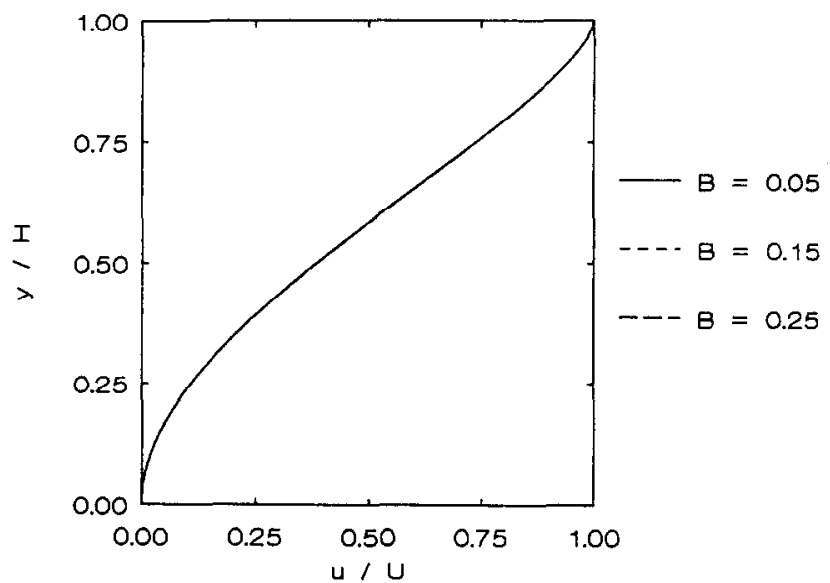

Fig. 2. Theoretical dimensionless velocity profile for $A=1.0$ for various values of the parameter $B$.

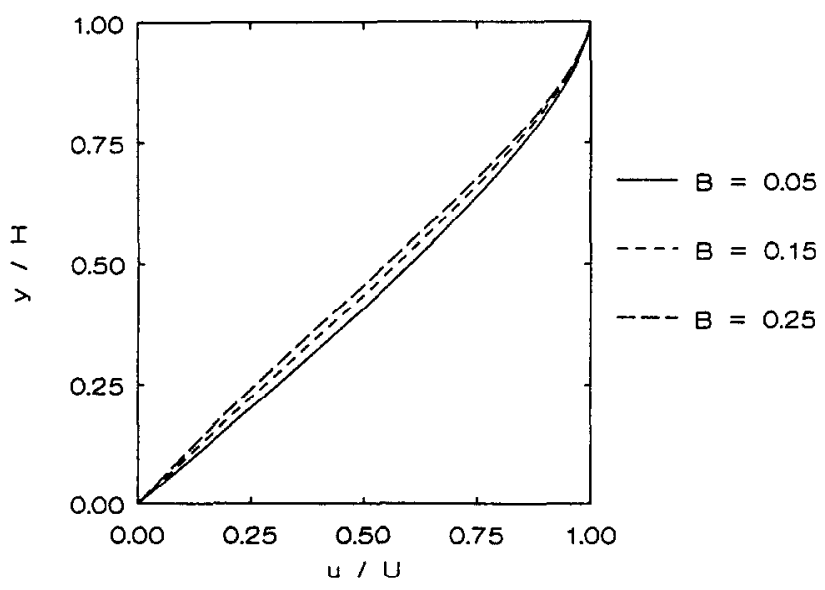

Fig. 3. Theoretical dimensionless velocity profile for $A=1.1$ for various values of the parameter $B$.

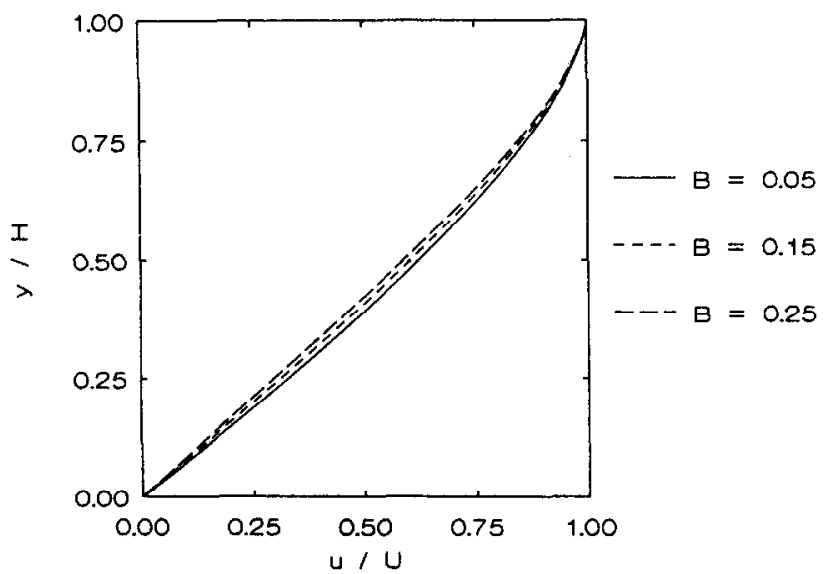

Fig. 4. Theoretical dimensionless velocity profile for $A=1.2$ for various values of the parameter $B$.

\section{Experimental}

The velocity profile of cohesionless granular materials flowing down inclined chutes has been studied exper- imentally by many authors: for example [3], [6], [12-18]. Only the studies where the flow was fully developed and no slip occurred on the base of the chute are relevant to this work, [3], $[14,15,17]$.

\section{Test set-up and experimental procedure}

The experiments were performed in a chute with a length of $500 \mathrm{~cm}$ and a width of $14 \mathrm{~cm}$. Its walls were made of glass plates, which were carefully aligned, to permit visual observation of the flow. The base of the chute was roughened with profiled rubber sheets; the direction of the profile was perpendicular to the direction of flow. In this way the no-slip condition at the base of the chute was enforced and a velocity gradient was created in the chute. The chute angle $\alpha$ was varied between $26.4^{\circ}$ and $38.1^{\circ}$.

The material used consisted of black plastic particles. The irregular particle shape can be described as cylinderlike, with a 'height' of $\sim 2 \mathrm{~mm}$ and a 'diameter' of $\sim 2.5 \mathrm{~mm}$. A small fraction of white plastic particles was added to function as a tracer. Quasi-static tests of the plastic particles in a Jenike shear tester gave a quasi-static friction angle $\phi_{\mathrm{s}}=38^{\circ}$, a wall friction angle $\epsilon=11^{\circ}$ and a density $\rho=630 \mathrm{~kg} \mathrm{~m}^{-3}$. Tests in annular shear tester resulted in $\phi_{\mathrm{s}}=35^{\circ}$.

The material was circulated in a continuous operating loop which allowed proper adjustment of the flow and eliminated starting-up effects. This loop consisted of a conveyor belt located at the outlet of the chute and a bucket elevator which delivered the material to a hopper from which the material flowed into the chute. The discharge rate into the chute could be regulated by varying the outlet of the hopper. The mass flow rate was measured by a weighing installation in the conveyor belt component of the loop.

A photographical method was employed to measure the velocity of the tracer particles through the glass side wall. In front of the camera a disc with eight equidistant holes in it rotated at a rate that was varied between 400 and $2200 \mathrm{rpm}$. During the fixed exposure time of $0.033 \mathrm{~s}$ the film was 'exposed' each time a hole was in front of the lens. This resulted in a photo showing several images of the same tracer particle at different points in time. From the known rate of rotation of the disc and the successive positions of the tracer particles, the velocity of the tracers was determined. Although this procedure was time-consuming, the velocities at the side wall could be measured accurately. The photographs were made at a distance of $113.5 \mathrm{~cm}$ upstream from the outlet of the chute.

An often used alternative to photographical methods is the fibre-optic method, see for example [14]. Disadvantages of the fibre-optic method for measuring the velocity are the influence of the separation distance between the probes and the sensitivity to the skewness 
of the probes with respect to the direction of flow. This last drawback is of importance when the exact flow direction is not known in advance. This is the case for immature sliding flow where a stagnant zone forms on the base of the chute.

The total height of the granular material, as well as the height of the stagnant zone if present, were determined visually at four different positions along the chute. From these observations the flow depth $H$ was detcrmined.

Electrostatic forces may be generated by the plastic particles moving along the glass side walls. No signs of electrical charge transfer, such as unusual dust accumulation or particles 'sticking' to the side wall, were ever observed.

Measurements were performed approximately $15 \mathrm{~min}$ after start-up of the flow in order to eliminate startingup effects.

\section{Flow type and flow depth}

The flow depth and the position of the zone of stagnant material (if present) were determined visually at four different positions along the chute for $\alpha=26.4^{\circ}$, $\alpha=30.8^{\circ}, \alpha=33.9^{\circ}$ and $\alpha=38.1^{\circ}$ for various mass flow rates. Immature sliding flow occurred, except for $\alpha=38.1^{\circ}$.

From these measurements, the angle $\gamma$ could be determined. The results are shown in Fig. 5. The results satisfy eqn. (12) reasonably well, except for the chute angle $\alpha=38.1^{\circ}$. This is in accordance with the developed theory, since for this chute angle fully developed sliding flow occurred.

A curve fit of eqn. (12) with the data for $\alpha=26.4^{\circ}$, $\alpha=30.8^{\circ}$ and $\alpha=33.9^{\circ}$ leads to $\phi_{\mathrm{s}}=31^{\circ}$ and $\epsilon=12^{\circ}$ for $k=1$. The value of $k \sim 1$ is in agreement with the theory of [19]. The values of $\phi_{\mathrm{s}}$ and $\epsilon$ determined from eqn. (12) are in reasonable agreement with the values as obtained with the Jenike shear tester and the annular shear tester.

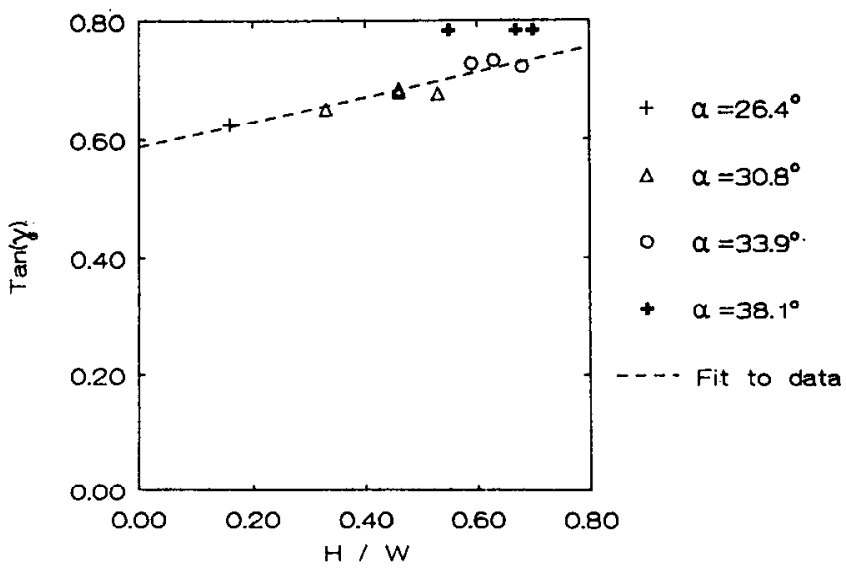

Fig. 5. Experimental results for $\tan \gamma$ vs. $H / B$ for various chute angles.

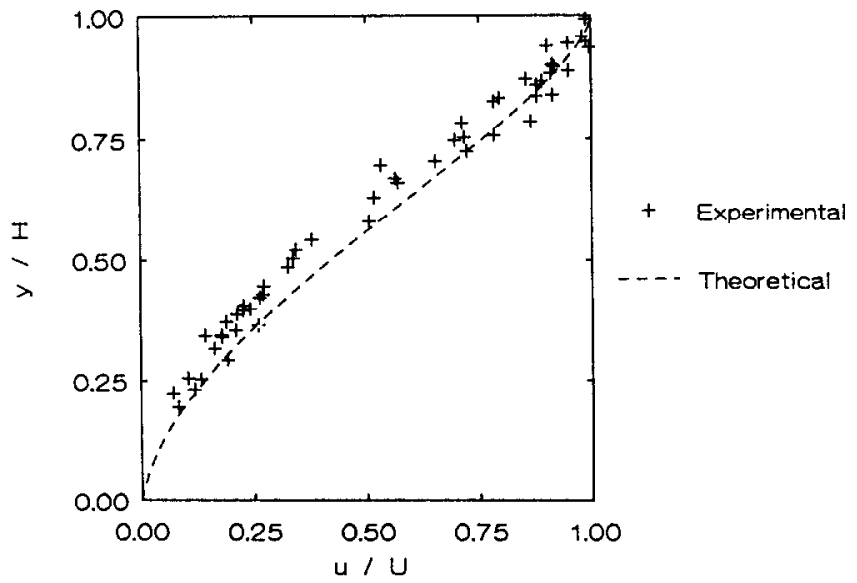

Fig. 6. Comparison between experimental and theoretical dimensionless velocity profile for chute angle $\alpha=30.8^{\circ}$.

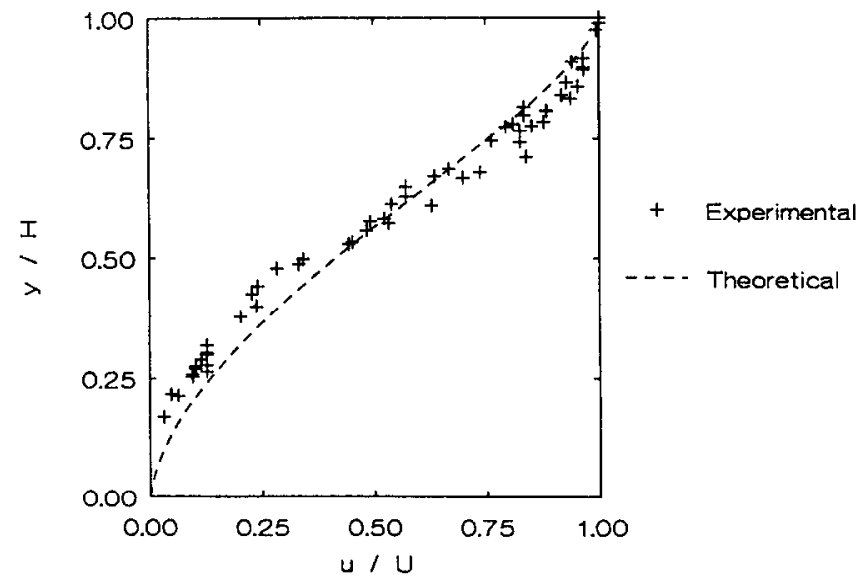

Fig. 7. Comparison between experimental and theoretical dimensionless velocity profile for chute angle $\alpha=33.9^{\circ}$.

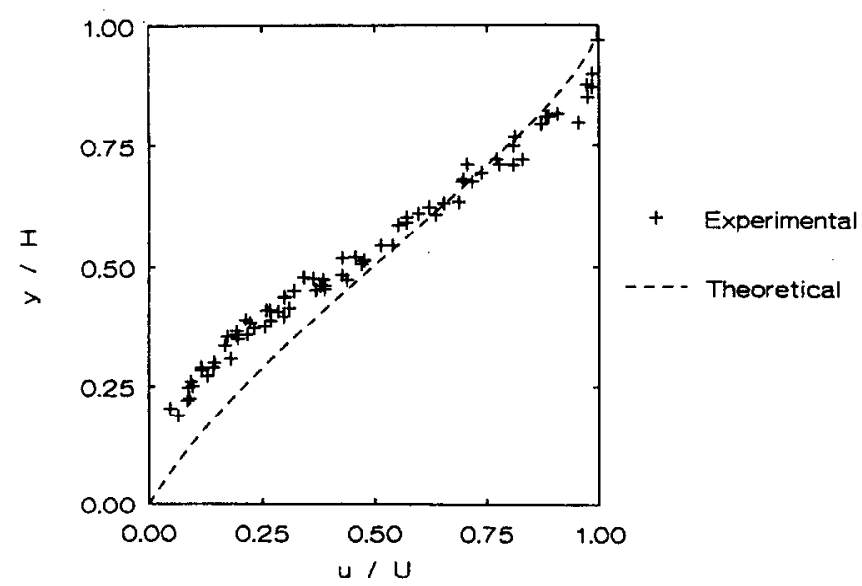

Fig. 8. Comparison between experimental and theoretical dimensionless velocity profile for chute angle $\alpha=38.1^{\circ}$.

\section{Velocity profiles}

Velocity profiles were measured for $\alpha=30.8^{\circ}, \alpha=33.9^{\circ}$ and $\alpha=38.1^{\circ}$. The dimensionless velocity profiles in the 
TABLE 1. Quantities associated with the measurements of the velocity profiles

\begin{tabular}{lllllll}
\hline $\begin{array}{l}\text { Chute } \\
\text { angle }\end{array}$ & $\begin{array}{l}Q \\
\left(\mathrm{~kg} \mathrm{~s}^{-1}\right)\end{array}$ & $\begin{array}{l}\text { Maximum } \\
\text { velocity } \\
\left(\mathrm{m} \mathrm{s}^{-1}\right)\end{array}$ & $\begin{array}{l}\text { Flow } \\
\text { depth } \\
(\mathrm{m})\end{array}$ & $\begin{array}{l}\text { Angle of } \\
\text { stagnant zone } \\
\text { with horizontal }\end{array}$ & $\begin{array}{l}\text { Average } \\
\text { density } \\
\left(\mathrm{kg} \mathrm{m}^{-3}\right)\end{array}$ & $A$ \\
\hline $30.8^{\circ}$ & 5.3 & 2.3 & 0.069 & $34.4^{\circ}$ & 605 & 1.00 \\
$33.9^{\circ}$ & 8.3 & 2.8 & 0.089 & $36.0^{\circ}$ & 560 & 0.17 \\
$38.1^{\circ}$ & 11.8 & 3.7 & 0.102 & not present & 555 & 1.00 \\
\hline
\end{tabular}

flowing region are given in Figs. 6, 7 and 8. Also shown are the theoretical predictions according to eqn. (24). The corresponding values of the parameters $A$ and $B$ according to eqns. (22) and (23) are given in Table 1.

The average density of the granular flowing material is computed from the measured velocity profile $u(y)$ and the measured mass flow rate $Q$ :

$Q=\rho W \int_{0}^{H} u(y) \mathrm{d} y$

It is noted that this method overestimates the density $\rho$, since the side wall friction decreases the velocity near the wall where it is measured.

Other relevant information is given in Table 1.

\section{Discussion}

A simple phenomenological theory has been developed of the flow of cohesionless granular materials down inclined chutes with a rough base. The theory includes dynamic stresses induced by interparticle collisions and quasi-static stresses induced by friction acting on semi-permanent interparticle contacts. These stresses are related in a nonlinear way, similar to the Casson model, to the total stress. A correction has been made in the determination of the stress field in the chute in order to account for the effect of the side wall friction. The theor predicts the existence of two flow types, immature sliding flow and fully developed sliding flow, depending on material properties, chute angle, mass flow rate, chute geometry and side wall friction. The velocity profile contains an inflection point for immature sliding flow, while for fully developed sliding flow the velocity profile becomes blunter.

The theoretical dimensionless velocity profile is in qualitative agreement with the observations of Savage [1] and the experimental results presented here.

The quantitative agreement between the measured and the theoretical dimensionless velocity profiles is reasonably good, especially for the chute angles $\alpha=30.8^{\circ}$ and $\alpha=33.9^{\circ}$. For the chute angle $\alpha=38.1^{\circ}$ the deviation is larger.
Although the stress field has been corrected for the presence of side wall friction, this has not been done for the velocity field. The side wall friction will decrease the velocity near the wall where it is measured. This is a possible explanation for the deviations between the theoretical and measured velocity profile. This effect is most prominent at higher chute angle and near the base of the flowing layer of granular material, since the wall friction will then be highest.

It was expected that the density would decrease when the chute angle was increased, since the shear rate increased too. The measured densities, as derived from the measured mass flow rate and the measured velocity profile near the wall, are given in Table 1 . As the chute angle was increased from $\alpha=33.9^{\circ}$ to $\alpha=38.1^{\circ}$ the density hardly changed, contrary to expectation. As noted before, the measured density is overestimated due to the slowing down of the material by the side wall friction. The small change in measured density between $\alpha=33.9^{\circ}$ to $\alpha=38.1^{\circ}$ and the deviation between theoretical and experimental velocity profile for $\alpha=38.1^{\circ}$ may both be caused by the side wall friction.

Whether or not the density changes over the height of the flowing material significantly affect the velocity profile is not clear. Since the purpose of this work was to develop a simple theory that predicts the two flow types and the associated velocity profile, no attempt has been made so far to include density variations in the theory. Experimental determination of density variations was not possible with the test set-up used.

The chute gcometry can affect the accuracy of the measurements in several ways. An important requirement is that the chute is sufficiently long in order that fully developed flow can occur. The chute used in this investigation was $500 \mathrm{~cm}$ long, while other investigators used (much) shorter chutes. The ratio of flow depth to chute width should be small, since otherwise side wall friction creates a variation of the velocity over the width of the chute. If the ratio of flow depth to particle radius is small, as in many previous investigations, it is doubtful whether a continuum-mechanical theory can describe the measurements, because in this case the flowing layer of material is formed by only few particles. 


\section{List of symbols}

$A$ coefficient defined by eqn. (22), -

$B$ coefficient defined by eqn. (23), -

$C$ coefficient defined by eqn. (21), $\mathrm{s}^{-1}$

$\mathrm{g}$ acceleration of gravity, $\mathrm{m} \mathrm{s}^{-2}$

$H$ flow depth of granular material, $\mathrm{m}$

$k \quad$ stress ratio between $T_{z z}$ and $T_{y y}$ at the side wall, -

$Q$ mass flow rate of granular material, $\mathrm{kg} \mathrm{s}^{-1}$

$T$ stress tensor with components $T_{x x}, T_{x y}$, etc., $\mathrm{N}$ $\mathrm{m}^{-2}$

$U$ velocity of granular material at top of flowing layer, $\mathrm{m} \mathrm{s}^{-1}$

$u \quad$ velocity of granular material, $\mathrm{m} \mathrm{s}^{-1}$

$W$ chute width, $\mathrm{m}$

$x \quad$ coordinate in the direction of flow, $\mathrm{m}$

$y$ coordinate perpendicular to the free surface, $m$

$z$ coordinate along the width of the chute, $m$

\section{Greek letters}

$\alpha \quad$ angle of chute with horizontal, -

$\beta$ angle of stagnant zone with horizontal, -

$\epsilon \quad$ friction angle of side wall, -

$\phi_{\mathrm{s}} \quad$ quasi-static friction angle of granular material, -

$\phi_{\mathrm{D}}$ dynamic friction angle of granular material, -

$\gamma \quad$ angle of flowing layer of granular material with horizontal, - $\mu \quad$ generalised viscosity in eqn. (15), $\mathrm{kg} \mathrm{m}^{-1}$

$\rho$ density of granular material, $\mathrm{kg} \mathrm{m}^{-3}$

\section{References}

1 S. B. Savage, Adv. Appl. Mech., 24 (1984) 289.

2 C. S. Campbell, Ann. Rev. Fluid Mech., 22 (1990) 57.

3 M. Ishida, T. Hatano and T. Shirai, Powder Technol., 27 (1980) 7.

4 M. Sayed and S. B. Savage, J. Appl. Math. Phys., 34 (1983) 84.

5 P. C. Johnson and R. Jackson, J. Fluid Mech., 176 (1987) 67.

6 P. C. Johnson, P. Nott and R. Jackson, J. Fluid Mech., 210 (1990) 501.

7 N. Casson, in C. C. Mill (ed.), Rheology of Disperse Systems, Pergamon Press, Oxford, 1959, p. 84.

8 R. A. Bagnold, Proc. $R$ Soc. London, Ser. A, 225 (1954) 49.

9 M. Sayed and S. B. Savage, J. Fluid Mech., 142 (1984) 391

10 D. M. Hanes and D. L. Inman, J. Fluid Mech., 150 (1985) 357.

11 K. Craig, R. H. Buckholz and G. Domoto, J. Appl. Mech., 53 (1986) 935.

12 D. A. Augenstein and R. Hogg, Powder Technol., 19 (1978) 205.

13 P. C. Knight, Powder Technol., 37 (1983) 183.

14 S. B. Savage, J. Fluid Mech., 92 (1979) 53.

15 M. Ishida and T. Shirai, J. Chem. Eng. Jpn., 12 (1979) 46.

16 H. Ahn, C. E. Brennen and R. H. Sabersky, J. Appl. Mech., 58 (1991) 792.

17 J. Bailard, Ph.D. Thesis, University of California, San Diego, 1978.

18 T. G. Drake, J. Fluid Mech., 225 (1991) 121.

19 S. B. Savage and D. J. Jeffrey, J. Fluid Mech., 110 (1981) 255. 